\title{
Le Managed Care permet d'éviter les cathéters cardiaques inutiles
}

\author{
Corinne Chmiela; Oliver Reich ${ }^{\mathrm{b}, \mathrm{c}}$ \\ ${ }^{a}$ Institut für Hausarztmedizin, Universität und Universitätsspital Zürich; b Groupe Helsana; ${ }^{c}$ santé24, SWICA Organisation de santé
}

Dans un contexte non urgent, les lignes directrices recommandent une prise en charge diagnostique non invasive de l'accident ischémique pour la majorité des patients chez lesquels on suspecte une maladie coronarienne. Néanmoins, chez une proportion substantielle de ces patients - en l'absence de prise en charge diagnostique non invasive de l'accident ischémique au préalable - une coronarographie invasive est effectuée afin d'établir un diagnostic sans qu'il en résulte pour autant une intervention thérapeutique.

\section{Une question de responsabilité des coûts et de coordination}

Quelle est l'influence des modèles d'assurance alternatifs sur le nombre de coronarographies inutiles par rapport à l'assurance de base standard (assurance obligatoire des soins, AOS)? Cette question a été examinée par l'Institut für Hausarztmedizin de Zurich en collaboration avec le groupe Helsana sur la base des données de l'assurance maladie.

\section{Une question de contrôle}

Dans un système de santé comme celui de la Suisse, où l'assurance maladie de base garantit le libre accès à tous les niveaux de soins de santé, il est difficile de coordonner une fonction de contrôle en vue d'optimiser les soins médicaux. Avec les modèles d'assurance alternatifs (MAA), la personne assurée accepte volontairement des restrictions en matière d'accès aux soins médicaux et bénéficie d'une réduction de prime en contrepartie. Les MAA ont des fonctions de régulation différentes qui peuvent en principe être divisées en deux groupes:

1. Les modèles de Gate Keeping (GK) avec des mécanismes de contrôle, tels que les modèles de télémédecine et les modèles de médecins de famille sans responsabilité budgétaire;

2. Le Managed Care (MC) - ou modèles de médecins de famille avec coresponsabilité budgétaire des médecins participants.

\section{Une question d'ordre}

L'étude [1] a analysé les données d'assurance de 11250 patients (>18 ans) ayant subi une coronarographie élective entre 2012 et 2015. Ont été prises en compte les coronarographies sans angioplastie simultanée/consécutive (pose de stents) ni intervention de chirurgie cardiaque - c.-à-d. des examens diagnostiques invasifs sans conséquences thérapeutiques. Les patients ayant subi un infarctus aigu du myocarde ou une intervention d'urgence ont été exclus. Il a été documenté si ces patients avaient bénéficié au préalable d'une prise en charge diagnostique non invasive appropriée, c'est-à-dire un ou plusieurs des examens suivants:

- ECG à l'effort;

- échocardiographie transthoracique au repos;

- échocardiographie en situation de stress;

- scintigraphie myocardique;

- tomodensitométrie cardiaque;

- imagerie par résonance magnétique du cœur.

\section{Une question de nécessité}

L'étude a montré que parmi les patients étudiés, 3044 $(33,2 \%)$ ont subi une coronarographie sans conséquence thérapeutique et sans examen préalable non-invasif. Comparativement à la population de patients de l'assurance de base standard, les patients assurés MC ont bénéficié beaucoup plus fréquemment de dépistages d'ischémie cardiaque non invasifs préa- 
lables (p <0,001; OR 1,17; IC à 95\%:1,045-1,312). Cette association est demeurée statistiquement significative de façon indépendante après correction pour les facteurs perturbateurs potentiels suivants: âge, sexe, assurance complémentaire, traitement hospitalier, médicaments cardiovasculaires, comorbidités chroniques, état de risque élevé (patients ayant subi une intervention cardiaque thérapeutique au cours du mois suivant ou des 18 mois précédant le diagnostic par coronarographie). Par rapport aux patients assurés $\mathrm{MC}$, aucune différence n'a été constatée chez ceux des modèles GK dans la proportion d'examens non invasifs par rapport aux patients assurés AOS ( $p=0,07$; adj. OR 1,11; IC à 95\%: 0,9911,253).

Cette étude montre que les modèles MC avec coordination par le médecin de famille et coresponsabilité des coûts conduisent à une réduction des prises en charge diagnostiques par coronarographie inappropriées par rapport à l'assurance de base standard avec accès illimité aux soins de santé ou également par rapport aux

\section{Cette étude montre que les modèles $\mathrm{MC}$ avec coordination par le médecin de famille et coresponsabilité des coûts conduisent à une réduction des prises en charge diagnostiques par coronarographie inappropriées.}

modèles GK sans responsabilité des coûts. Un statut d'assurance MC peut donc aider à prévenir les risques potentiels d'interventions inutiles. Le fait que les patients assurés MC subissaient plus fréquemment des tests non invasifs réfute pareillement l'hypothèse selon laquelle la coresponsabilité du corps médical à l`égard des coûts chez des patients assurés MC entraîne une sous-utilisation des services de santé ou une diminution des examens diagnostiques.

\section{Une question de cause}

Létude ne répond pas à la question de savoir pourquoi les patients assurés MC bénéficient d'un processus diagnostique plus adéquat. Une explication possible est que les modèles d'assurance MC ne sont proposés que dans les réseaux des médecins de famille. Ces réseaux s'engagent à respecter les mesures internes d'assurance qualité et de promotion de la qualité. Les mécanismes de contrôle et de régulation au sein de ces réseaux visent à accroître l'adhérence de leurs membres aux recommandations fondées sur des preuves.

\section{Une question d'avenir}

La question de savoir s'il existe d'autres mécanismes de contrôle que le MC, afin d'offrir une prise en charge plus adaptée aux patients, fait actuellement l'objet de recherches dans le domaine des soins de santé. A titre d'exemple, l'initiative Choosing Wisely a lancé une campagne pour informer le public que les coronarographies inutiles étaient l'un de ses principaux points d'attaque. Par conséquent, les compagnies d'assurance ont inclus la pertinence de la coronarographie dans leurs systèmes de rémunération Pay-for-Performance, avec ces interventions sont moins bien payées si les critères ne sont pas respectés. Cette combinaison de la mise en œuvre de registres et des initiatives de qualité a été si efficace que le taux de coronarographie inadéquate a été réduit de $26,6 \%$ [2]. Reste à savoir si une telle approche pourrait également être mise en œuvre en Suisse. En tout état de cause, il reste intéressant d'observer l'évolution de la discussion sur les coûts et la qualité en Suisse. De telles études fournissent une base scientifique valable pour les discussions politiques et pour l'élaboration de solutions constructives.

\section{Références}

1 Chmiel C, Reich O, Signorell A, et al. Effects of managed care on the proportion of inappropriate elective diagnostic coronary angiographies in non-emergency patients in Switzerland: a retrospective crosssectional analysis. BMJ Open 2018;8:e020388. doi:10.1136/bmjopen-2017-020388.

2 Desai N, Bradley S, Parzynski C, et al. Appropriate Use Criteria for Coronary Revascularization and Trends in Utilization, Patient Selection, and Appropriateness of Percutaneous Coronary Intervention - FindingsFrom the NCDR- CathPCI Registry Circulation 2015;132:2278-78. 